Reprod. Nutr. Dévelop., 1980, 20 (4 B), 1309-1316.

\title{
Influence de l'apport et du mode d'administration de triglycérides sur l'absorption et l'hydrolyse des phosphatidylcholines de bile chez le rat
}

\author{
par Micheline BOQUILLON, Ph. BOUCROT
}

avec la collaboration technique de Monique CHABIN, Marguerite-Marie BOUTILLON et J. GRESTI

Loboratoire de Physiologie onimale el de la Nutrition, Sciences Mirande, B. P. 138, 21004 Dijon Cedex.

Summary. Effect of amount and mode of triglyceride administration on the absorption and the hydrolysis rates of rat native bile phosphatidylcholine.

The paper reports how exogenous triglycerides, present in the intestinal lumen, modify the hydrolysis rates of bile phosphatidylcholine and the absorption of non-hydrolysed and hydrolysed bile lipids. The exogenous triglyceride was either given in a meal (experiment A) or injected into the intestinal lumen (experiment B).

Experiment A : Group $E_{1}$ received a meal with $480 \mathrm{mg}$ of olive oil added with a tracer dose of tri $\left(1-{ }^{14} \mathrm{C}\right)$ oleoyl glycerol. Group $\mathrm{T}_{1}$ received a similar meal but without fat. Two hours later, the rats received in the duodenum $0.1 \mathrm{ml}$ of bile $(86.5 \mathrm{p} .100$ of the radioactivity of the phosphatidylcholine labelled with $\left(9-10^{3} \mathrm{H}_{2}\right)$ oleic acid) ; they were killed 90 min later. In the intestinal lumen, small amounts of exogenous and endogenous lipids $(<20 \mathrm{mg})$ were found ; they changed neither the bile lipid absorption (70-75 p. 100 of the radioactive dose disappeared from the lumen) nor the hydrolysis rate of bile phosphatidylcholine which remained the main radioactive lipid of the intestinal lumen and mucosa.

Experiment $B$ : The rats were fitted with a bile and a lymph fistula. Group $E_{2}$ received 6 injections in the duodenum at 1-hour intervals; each injection (one per hour) contained $80 \mathrm{mg}$ of trioleoyl glycerol and $0.5 \mathrm{ml}$ of bile $(93 \mathrm{p} .100$ of the radioactivity of the phosphatidylcholine labelled with $\left(2-{ }^{3} \mathrm{H}\right)$ glycerol and $\left(1-{ }^{14} \mathrm{C}\right)$ palmitic acid). Group $\mathrm{T}_{2}$ received only the bile samples. The rats were killed half an hour after the 6th injection. The high quantities of exogenous triglyceride, fatty acid and partial glyceride (>200 mg) in the intestinal contents of group $\mathrm{E}_{2}$ increased slightly bile lipid hydrolysis and absorption ; the bile phosphatidylcholine appeared to be the main lipid of the intestinal lumen and mucosa. From the mucosa to the lymph the ${ }^{3} \mathrm{H}-{ }^{14} \mathrm{C}$ of the phosphatidylcholine did not change.

It was concluded that the hydrolysis and absorption rates of rat native bile phosphatidylcholine were highly independent of the amounts of exogenous triglycerides, fatty and partial glyceride present in the infestinal lumen : less than $20 \mathrm{mg}$ (experiment $\mathrm{A}$ ) or an overload dose (>200 mg) never found after a fat meal (experiment B).

\section{Introduction.}

L'influence des lipides exogènes sur l'hydrolyse et l'absorption des phosphatidylcholines biliaires chez le rat a fait l'objet d'une seule étude in vivo (Reisser e Boucrof, 1978) dans laquelle on introduit directement dans le duodénum, en $30 \mathrm{sec}$, un échan- 
tillon de bile marquée par l'acide $\left(9-10^{3} \mathrm{H}_{2}\right)$ oléique additionnée de quantités variables de trioleoyl glycérol. Lorsque les quantités de trioleoyl glycérol étaient importantes (30 mg) l'absorption et l'hydrolyse des phosphatidylcholines biliaires étaient augmentées, tandis que lorsqu'elles étaient faibles ou nulles, l'intégrité de la molécule de phosphatidylcholine biliaire était respectée car les acides gras insaturés libérés de la position 2 du glycérol, étaient en faibles quantités.

II nous a paru intéressant de reprendre une telle étude en nous plaçant dans des conditions plus physiologiques. Aussi, dans les expériences A, on introduit dans l'estomac un repas qui comporte $480 \mathrm{mg}$ d'huile d'olive additionnée d'une dose traceuse de tri $\left(1-{ }^{14} \mathrm{C}\right)$ oleoyl glycérol, puis dans le duodénum, un faible volume de bile $(0,1 \mathrm{ml})$ marquée à l'acide $\left(9-10^{3} \mathrm{H}_{2}\right)$ oléique, de manière à ne pas surcharger la lumière infestinale en matériel biliaire qui est estimé à l'équivalent de $0,5 \mathrm{ml}$ de bile environ (Nalbone et al., 1974 ; Boucrot, 1979). Nous avons voulu comparer les expériences A à des expériences $B$ dans lesquelles les rats sont porteurs d'une fistule biliaire et d'une fistule lymphatique ; on introduit dans le duodénum, par 6 injections échelonnées sur $5 \mathrm{~h}$ une surcharge de lipides exogènes (au total $480 \mathrm{mg}$ de trioleoyl glycérol) et de la bile doublement marquée $\left[\left(2-{ }^{3} \mathrm{H}\right)\right.$ glycérol et acide $\left(1-{ }^{14} \mathrm{C}\right)$ palmitique] qui compense exactement le volume de bile dérivée hors de l'organisme par la fistule biliaire.

Le marquage de la bile a été choisi de façon qu'on puisse suivre dans les expériences $A$ les phosphatidylcholines non hydrolysées ou les acides gras insaturés libérés de la position 2 du glycérol, et dans les expériences B, les lysophosphatidylcholines.

\section{Matériel et méthodes.}

\section{Obfention des triglycérides ef de la bile.}

Toutes les molécules marquées sont pures à 98 p. 100 et sont fournies par le CEA (Saclay, F) sauf le $\left(2-{ }^{3} \mathrm{H}\right)$ glycérol (Amersham, GB). Dans les expériences $A$, le tri $\left(1-{ }^{14} \mathrm{C}\right)$ oleoyl glycérol, d'activité spécifique $51 \mathrm{mCi} / \mathrm{mmole}$ est ajouté sous forme de traceur à $480 \mathrm{mg}$ d'huile d'olive. Cette huile comportant 35 p. 100 de ses triglycérides sous forme de tri-oleoyl glycérol (Descargues, 1975), l'activité spécifique finale du $\operatorname{tri}\left(1-{ }^{14} \mathrm{C}\right)$ oleoyl glycérol est $0,019 \mathrm{mCi} / \mathrm{mmole}$.

- Bile marquée à l'acide $\left(9-10^{3} \mathrm{H}_{2}\right)$ olélque : $2 \mathrm{ml}$ de bile radioactive sont recueillis en $3 \mathrm{~h}$, chez un rat porteur d'une fistule biliaire qui a reçu par la veine mésentérique $0,5 \mathrm{mCi}$ de $\left(9-10^{3} \mathrm{H}_{2}\right)$ oléate de potassium d'activité spécifique $128 \mathrm{mCi} / \mathrm{mmole}$ fixé sur sérumalbumine. L'activité spécifique des phosphatidylcholines biliaires est $0,68 \mathrm{mCi} / \mathrm{mmole} .86,5$ p. 100 de la radioactivité lipidique $\left({ }^{3} \mathrm{H}\right)$ sont portés par les phosphatidylcholines et 13,5 p. 100 par les autres lipides.

- Bile marquée au $\left(2-{ }^{3} \mathrm{H}\right)$ glycérol ef à l'acide $\left(1-{ }^{14} \mathrm{C}\right)$ palmitique : chaque rat reçoit, par la veine mésentérique, $0,25 \mathrm{mCi}$ de $\left(1-{ }^{14} \mathrm{C}\right)$ palmitate de potassium d'activité spécifique $28,6 \mathrm{mCi} / \mathrm{m}$ mole fixé sur sérum albumine, et $1,7 \mathrm{mCi}$ de $\left(2-{ }^{3} \mathrm{H}\right)$ glycérol d'activité spécifique $134 \mathrm{mCi} / \mathrm{mmole}$. Plusieurs échantillons de bile collectée pendant $2,5 \mathrm{~h}$ sont réunis. L'activité spécifique finale des phosphatidylcholines du mélange de biles est pour le $\left({ }^{14} \mathrm{C}\right): 0,2 \mathrm{mCi} / \mathrm{mmole}$ et pour le $\left({ }^{3} \mathrm{H}\right): 0,33 \mathrm{mCi} / \mathrm{mmole}$. La répartition de la radioactivité entre les constituants lipidiques est, pour le $\left({ }^{14} \mathrm{C}\right)$ et le $\left({ }^{3} \mathrm{H}\right)$ : phosphatidylcholines : 93 p. 100, autres lipides : 7 p. 100. 
Les échantillons de bile contiennent en moyenne : $2,71 \pm 0,24 \mathrm{mg}$ de phosphatidylcholines par $\mathrm{ml}$ de bile. La bile est utilisée dans un délai de $48 \mathrm{~h}$.

Profocole expérimental.

Expériences $A$ : Ingestion d'un repas qui comporte des triglycérides. - Des rats Wistar de $190 \mathrm{~g}$ subissent un jeûne hydrique de $15 \mathrm{~h}$. Un lot $\mathrm{E}_{1}$ reçoit par intubation gastrique un repas qui comprend $480 \mathrm{mg}$ d'huile d'olive additionnée de $3,5 \mu \mathrm{Ci}$ de tri- $\left(1-{ }^{14} \mathrm{C}\right)$ oleoyl glycérol en dose traceuse, 1,5 g de lactose, 1,2 g d'hydrolysat de caséine et $5 \mathrm{ml}$ d'eau ; les lipides sont émulsifiés par ultrasons (sonde de $40 \mathrm{~W}$ pendant $30 \mathrm{sec}$.). Un lot témoin $T_{1}$ reçoit le repas sans lipides. Deux heures plus tard, on injecte à tous les rats, anesthésiés à l'éther éthylique, dans le duodénum, $0,1 \mathrm{ml}$ de bile marquée à l'acide $\left(9-10^{3} \mathrm{H}_{2}\right)$ oléique $(0,33 \mu \mathrm{Ci})$. Les rats sont sacrifiés par décapitation $90 \mathrm{~min}$ plus tard.

Expériences B : Introduction des triglycérides directement dans le duodénum. - Les rats, placés dans des cages de contention, sont porteurs à la fois d'une fistule biliaire et d'une fistule lymphatique thoracique. Seuls les animaux dont la lymphe s'est écoulée dans de bonnes conditions ont été retenus (volume moyen : $1,04 \pm 0,08 \mathrm{ml} / \mathrm{h}$ de collecte). Un lot $E_{2}$ reçoit, par injection dans le canal pancréato-biliaire cathétérisé, toutes les heures, $80 \mathrm{mg}$ de trioleoyl glycérol et $0,5 \mathrm{ml}$ de bile marquée par le $\left(2-{ }^{3} \mathrm{H}\right)$ glycérol et l'acide $\left(9-10^{3} \mathrm{H}_{2}\right)$ palmitique. Un lof témoin $T_{2}$ ne reçoil que la bile. Tous les rałs sont sacrifiés une demi-heure après la sixième injection. De cette façon, le volume de bile sécrétée vers l'extérieur est compensé par celui qui est injecté vers le duodénum.

Dans toutes les expériences, les contenus de l'estomac et de l'intestin grêle (du pylore jusqu'au cæcum) sont recueillis par lavage $(2 \times 20 \mathrm{ml})$ avec du sérum physiologique additionné de $3 \mathrm{~g} / \mathrm{l}$ de lauroglycocholate de sodium (Sigma, USA). La muqueuse de l'intestin grêle est grattée à $4^{\circ} \mathrm{C}$ à l'aide d'une spatule de verre.

Analyses : Les lipides de tous les échantillons (bile, contenu et muqueuse de l'intestin, lymphe) sont extraits selon la technique de Folch, Lees et Sloane-Stanley (1957). Les constituants lipidiques sont séparés par chromatographie sur couches minces (Reisser et Boucrot, 1978). Les mesures de radioactivité par scintillation liquide sont effectuées après avoir ajouté au silicagel $1 \mathrm{ml}$ d'eau distillée et $10 \mathrm{ml}$ de liquide scintillant (triton X100/butyl PBD à $12 \mathrm{~g} / /$ de xylène, $1 / 2 \mathrm{v} / \mathrm{v}$ ).

Les masses des acides gras des constituants lipidiques ont été évaluées par chromatographie en phase gazeuse à l'aide d'un standard interne : l'acide C17 : 0.

\section{Résulfats.}

Expériences $A(t a b l .1)$ : sacrifice 3 h 30 après la prise du repas.

Dans le lot $E_{1}$ on ne retrouve dans l'estomac que 24,3 p. 100 de la masse de tri $\left(1-{ }^{14} \mathrm{C}\right)$ oleoyl glycérol initialement introduite dans l'estomac et 3,2 p. 100 dans le contenu de l'intestin grêle ; à ce niveau, la répartition de la radioactivité entre les lipides montre que le $\operatorname{tri}\left(1-{ }^{14} \mathrm{C}\right)$ oleoyl glycérol a été hydrolysé. Au contraire, dans les deux lots $E_{1}$ et $T_{1}$, les phospholipides marqués au $\left({ }^{3} \mathrm{H}\right)$ sont peu hydrolysés : il apparaît 
TABLEAU 1

Expériences $\mathrm{A}$ : radioactivité $\left({ }^{3} \mathrm{H}\right)$ ef $\left({ }^{14} \mathrm{C}\right)$ des lipides des contenus de l'estomac et de l'intestin grêle et de la muqueuse intestinale (p. 100 de la dose administrée) ef masses des glycérides du contenu de l'intestin grêle (mg d'acides gras). Les rats $E_{1}$ ont reçu le repas qui comporte des lipides (dont le tri $\left({ }^{1}-{ }^{14} \mathrm{C}\right)$ oleoylglycérol), les rats $T_{1}$, le repas sans lipides. $2 \mathrm{~h}$ plus tard, tous les rats reçoivent une injection intraduodénale de $0,1 \mathrm{ml}$ de bile marquée à l'acide $\left(9-10^{3} \mathrm{H}_{2}\right) 18: 1$. Ils sont sacrifiés $90 \mathrm{~min}$ plus fard.

\begin{tabular}{|c|c|c|c|c|c|c|c|}
\hline \multirow{3}{*}{$\begin{array}{l}\text { Constiłuants } \\
\text { lipidiques }\end{array}$} & \multirow{3}{*}{$\begin{array}{l}\text { Lots } \\
\text { de } \\
\text { rats }\end{array}$} & \multirow{3}{*}{$\begin{array}{c}\text { Estomac } \\
\text { (contenu) } \\
\left({ }^{3} \mathrm{H}\right) \quad\left({ }^{4} \mathrm{C}\right)\end{array}$} & \multicolumn{5}{|c|}{ Intestin grêle } \\
\hline & & & \multicolumn{2}{|c|}{ Contenu } & \multirow{2}{*}{$\begin{array}{c}\text { Masses (mg } \\
\text { d'acides } \\
\text { gras) }\end{array}$} & \multicolumn{2}{|c|}{ Muqueuse } \\
\hline & & & $\left({ }^{3} \mathrm{H}\right)$ & $\left({ }^{14} \mathrm{C}\right)$ & & $\left({ }^{3} \mathrm{H}\right)$ & $\left({ }^{14} \mathrm{C}\right)$ \\
\hline $\begin{array}{l}\text { Lipides } \\
\text { totaux }\end{array}$ & $\begin{array}{l}T_{1} \\
E_{1}\end{array}$ & $24,3 \pm 2,6$ & & & & $\begin{array}{l}15,0 \pm 3,1 \\
18,4 \pm 3,8\end{array}$ & $0,7 \pm 0,3$ \\
\hline Phospholipides & $\begin{array}{l}T_{1} \\
E_{1}\end{array}$ & & $\begin{array}{l}17,7 \pm 5,8 \\
16,0 \pm 5,9\end{array}$ & $0,1 \pm 0,0$ & nd & & \\
\hline $\begin{array}{l}\text { Glycérides } \\
\text { partiels }\end{array}$ & $\begin{array}{l}T_{1} \\
E_{1}\end{array}$ & & $\begin{array}{l}2,8 \pm 0,8 \\
1,7 \pm 0,7\end{array}$ & $0,9 \pm 0,4$ & $\begin{array}{l}0,5 \pm 0,1 \\
1,7 \pm 0,4 *\end{array}$ & & \\
\hline Acides gras & $\begin{array}{l}T_{1} \\
E_{1}\end{array}$ & & $\begin{array}{l}3,1 \pm 0,9 \\
1,6 \pm 0,6\end{array}$ & $0,8 \pm 0,4$ & $\begin{array}{l}2,6 \pm 0,7 \\
5,9 \pm 1,3\end{array}$ & & \\
\hline Triglycérides & $\begin{array}{l}T_{1} \\
E_{1}\end{array}$ & & $\begin{array}{l}1,1 \pm 0,2 \\
0,3 \pm 0,2\end{array}$ & $1,3 \pm 0,5$ & $\begin{array}{l}2,0 \pm 1,0 \\
6,1 \pm 1,3 *\end{array}$ & & \\
\hline
\end{tabular}

Moyennes \pm écart standard à la moyenne ; ${ }^{*}$ significatif à $P<0,05 ;$ nd $=$ non déterminé ; $\mathrm{T}_{1}=4$ rats $; \mathrm{E}_{1}:=5$ rats.

peu d'acides gras marqués au $\left({ }^{3} \mathrm{H}\right)$, susceptibles d'être libérés de la position 2 du glycérol des phosphatidylcholines par la phospholipase $A_{2}$. II n'y a pas de différence significative entre les lots $T_{1}$ et $E_{1}$, lorsqu'on considère la disparition des lipides biliaires du contenu intestinal (environ les $3 / 4$ de la dose radioactive) ou l'apparition de lipides radioactifs dans la muqueuse (environ le sixième de la dose radioactive). Dans les deux lots, les masses d'acides gras, de glycérides partiels et de triglycérides intraluminaires sont faibles (moins de $20 \mathrm{mg}$ au total), mais elles sont significativement augmentées dans le lot $E_{1}$ par rapport au lot $T_{1}$.

Les lipides marqués au ${ }^{14} \mathrm{C}$ (ayant pour origine les triglycérides du repas) franchissent rapidement la muqueuse puisqu'ils ne supportent que 0,7 p. 100 de la dose radioactive introduite dans l'estomac.

\section{Expériences B (fabl. 2).}

A. - Contenu de l'intestin grêle : Dans le lot $\mathrm{T}_{2}$, les lipides biliaires sont peu hydrolysés : les phosphatidylcholines portent 36,1 p. $100 \mathrm{du}\left({ }^{3} \mathrm{H}\right)$ et 34,8 p. $100 \mathrm{du}\left({ }^{14} \mathrm{C}\right)$ de la dose radioactive injectée dans le duodénum, alors que la radioactivité des lipides totaux marqués au $\left({ }^{3} \mathrm{H}\right)$ s'élève à 44,3 p. 100 et celle marquée au $\left({ }^{14} \mathrm{C}\right)$ à 48,2 p. 100 ; leur rapport $\left({ }^{3} \mathrm{H}\right) /\left({ }^{14} \mathrm{C}\right)$ ne varie pas. Les lysophosphatidylcholines marquées au $\left({ }^{3} \mathrm{H}\right)$ et au $\left({ }^{14} \mathrm{C}\right)$ représentent le quart des phosphatidylcholines intraluminaires. On voit apparaître des acides gras marqués au $\left({ }^{14} \mathrm{C}\right)$ [L'acide $\left(1-{ }^{14} \mathrm{C}\right)$ palmitique se situe 




Reproduction, nutrition, développement 
en effet préférentiellement en position 1 dans les molécules de phosphatidylcholines, mais on en trouve en faible proportion en position 2 (Olsson et Boucrot, 1974 ; Boucrot, 1979)]. Dans le lot $\mathrm{E}_{2}$, le taux d'absorption des lipides biliaires est significativement augmenté du fait des injections intraluminaires de trioleoyl glycérol puisqu'on ne retrouve que 21,1 p. $100 \mathrm{du}\left({ }^{3} \mathrm{H}\right)$ et 18,3 p. $100 \mathrm{du}\left({ }^{14} \mathrm{C}\right)$ sur les phosphatidylcholines dont le rapport $\left({ }^{3} \mathrm{H}\right) /\left({ }^{14} \mathrm{C}\right)$ est élevé : 1,2 ; les lysophosphatidy t̂́cholines sont moins représentées que dans le lot $T_{2}\left(4,6\right.$ p. 100 contre 8,5 p. 100); $P<0,01$ pour le $\left({ }^{14} \mathrm{C}\right)$. Dans le lot $E_{2}$ les quantités de triglycérides $(143,2 \mathrm{mg})$, d'acides gras $(37,4 \mathrm{mg})$ et de monoglycérides $(27,5 \mathrm{mg})$ intraluminaires sont très élevées par rapport à celles trouvées dans le lot $T_{2}$.

B. - Muqueuse de l'intestin grêle : Dans les 2 lots, les lipides supportent entre 29,7 et 35,9 p. 100 de la radioactivité injectée initialement ; il n'y a pas de différence significative de la répartition de la radioactivité $\left({ }^{3} \mathrm{H}\right)$ et $\left({ }^{14} \mathrm{C}\right)$ entre les constituants : les phosphatidylcholines sont très marquées et ont un rapport $\left({ }^{3} \mathrm{H}\right) /\left({ }^{14} \mathrm{C}\right)$ qui s'élève à 1,2 (lot $T_{2}$ ) et à 1,4 (lot $E_{2}$ ). Les triglycérides sont assez marqués et ont, au contraire un rapport $\left({ }^{3} \mathrm{H}\right) /\left({ }^{14} \mathrm{C}\right.$ ) faible : 0,5 (lot $\mathrm{T}_{2}$ ) ef 0,7 (lot $\mathrm{E}_{2}$ ). Les autres constituants lipidiques sont peu marqués et n'ont pas été mentionnés sur le tableau.

C. - Lymphe : Les lipides de la lymphe sont peu marqués puisque plus de 50 p. 100 des lipides biliaires n'ont pas encore quitté l'intestin. Dans les 2 lots, les constituants lipidiques les plus marqués sont les triglycérides dont le rapport $\left({ }^{3} \mathrm{H}\right) /\left({ }^{14} \mathrm{C}\right)$ est assez proche de celui des triglycérides de la muqueuse ; les phosphatidylcholines sont aussi très marquées et ont un rapport $\left({ }^{3} \mathrm{H}\right) /\left({ }^{14} \mathrm{C}\right)$ qui est similaire à celui des phosphatidylcholines de la. muqueuse intestinale.

La radioactivité des lipides du contenu du cæcum et du gros intestin est très faible.

\section{Discussion.}

Il avait déjà été montré que dans la lumière intestinale, chez le Rat, en présence de lipides exogènes, les phosphatidylcholines de la bile sont prépondérantes par rapport à leurs produits d'hydrolyses : acides gras et lysophosphatidylcholines (Boucrot, 1979) et sont trouvées aussi bien dans la phase huileuse que dans la phase micellaire de la lumière de l'intestin grêle (Nalbone ef al., 1974 ; Boucrot, 1979). Dans les expériences $A$ et $B$ on a examiné l'influence des lipides exogènes sur l'hydrolyse des phosphatidylcholines de la bile et sur leur absorption par la muqueuse intestinale selon 2 protocoles très différents : dans l'expérience $A$ les lipides exogènes parviennent dans la lumière intestinale après l'ingestion d'un repas ; dans l'expérience $B$, par contre, on provoque une importante surcharge en lipides exogènes de la lumière intestinale, surcharge qui n'est évidemment jamais rencontrée lors de l'ingestion d'un repas.

Les résultats des deux tableaux monirent d'une part, que les phosphatidylcholines d'origine biliaire trouvées dans la lumière intestinale au moment du sacrifice sont faiblement hydrolysées et confirment d'autre part les travaux antérieurs (Olsson et Boucrot, 1974 ; Ulrich, Boucrot et Clément, 1974). En outre, on note l'intégrité des molécules de phosphatidylcholines biliaires même lorsque des triglycérides intraluminaires d'origine exogène (lot $E_{2}$ ) sont en forte quantités (143 mg). 
Après l'ingestion d'un repas sans lipides (lot $T_{1}$ ), la masse des lipides endogènes intraluminaires est faible : $11 \mathrm{mg}$ (valeur proche de celle indiquée par Raghavan et Ganguly (1967) chez le rat à jeun). Après l'ingestion du repas qui comporte (lot $E_{1}$ ) $480 \mathrm{mg}$ d'huile (dont $160 \mathrm{mg}$ de tri $\left(1-{ }^{14} \mathrm{C}\right.$ ) oleoyl glycérol), les lipides exogènes intraluminaires ne représentent, sous forme de triglycérides, de glycérides partiels et acides gras marqués au $\left({ }^{14} \mathrm{C}\right.$ ) que 3,1 p. 100 seulement (c'est-à-dire quelques $\mathrm{mg}$ ), de la dose radioactive introduite dans l'estomac, mais il s'ajoute à ceux-ci la masse des lipides endogènes intraluminaires (au total : $13 \mathrm{mg}$ environ). Les lipides marqués au $\left({ }^{14} \mathrm{C}\right.$ ) absorbés ne s'accumulent pas dans la muqueuse intestinale $(0,7$ p. 100 de la dose radioactive) ; de plus, 75 p. 100 de la dose radioactive au moment du sacrifice ont déjà quitté le tractus digestif. On note que l'ingestion du repas lipidique (lot $E_{1}$ ) n'accélère pas l'absorption des phosphatidylcholines biliaires radioactives. Selon Bennett Clark (1979), la muqueuse intestinale du rat intact serait capable d'absorber, par heure (après la prise d'un repas qui comporte des lipides) jusqu'à $160 \mathrm{mg}$ de lipides exogènes. Dans le lot $E_{2}$, des expériences $B$, la masse de trioleoyl glycérol introduite par injections répétées dans la lumière intestinale, n'atteint que $80 \mathrm{mg} / \mathrm{h}$, mais dans ce cas cette façon de procéder est peu physiologique, puisque lorsque le pylore joue son rôle, environ $13 \mathrm{mg}$ seulement de lipides (exprimés par leurs acides gras) sont retrouvés dans la lumière de l'intestin grêle des rats du lot $E_{1}$ (expérience $A$ ), alors que le repas comportait $480 \mathrm{mg}$ d'huile d'olive et qu'au moment du sacrifice, il en restait encore le 1/4 dans l'estomac. L'intérêt des expériences $B$ a été d'examiner le comportement des phosphatidylcholines de la bile lorsque la phase huileuse de triglycérides intraluminaires atteint des valeurs aussi élevées que celles trouvées dans la lumière intestinale des rats du lot $\mathrm{E}_{2}$. Ces phosphatidylcholines participent à l'hydrolyse des triglycérides exogènes et à l'absorption des grandes quantités de monoglycérides et d'acides gras puisque leur disparition du contenu intestinal dans le lot $E_{2}$ (76,9 p. 100 de la radioactivité injectée) est un peu plus élevée que dans le lot $T_{2}$ $(62,3$ p. 100). Toutefois, la différence esł faible, si on considère qu'au cours de l'expérience, environ la moitié (soit $240 \mathrm{mg}$ ) du trioleoyl glycérol injecté dans la lumière intestinale a été hydrolysé puis absorbé par les entérocytes. Les phosphatidylcholines représentent toujours les lipides les plus marqués des muqueuses intestinales des rats des lots $E_{2}$ ef $T_{2}$; leur rapport $\left({ }^{3} \mathrm{H}\right) /\left({ }^{14} \mathrm{C}\right)$ est plus élevé que celui des phosphatidylcholines intraluminaires ce qui indique que des remaniements ont eu lieu dans les entérocytes. II y a très peu de lysophosphatidylcholines marquées ce qui signifie que le lavage de la lumière intestinale a été efficace. La contribution des phosphatidylcholines d'origine biliaire à la formation de phosphatidylcholines lymphatiques est assez importante dans les deux lots. On trouve le même rapport $\left({ }^{3} \mathrm{H}\right) /\left({ }^{14} \mathrm{C}\right)$ entre les phosphatidylcholines de la muqueuse ef celles de la lymphe, mais une partie des phosphatidylcholines biliaires a été totalement hydrolysée ef le $\left(2-{ }^{3} \mathrm{H}\right)$ glycérol ef l'acide $\left(1-{ }^{14} \mathrm{C}\right)$ palmitique sont incorporés dans les triglycérides lymphatiques.

On peut conclure que malgré des protocoles expérimentaux très différents, les quantités variables de triglycérides exogènes présentes dans la lumière intestinale, influencent peu l'hydrolyse ef l'absorption des phosphatidylcholines de la bile. 
Remerciements. - Cette étude a bénéficié de l'appui financier de la Délégation générale à la recherche scientifique (France) contrat no 77.7.0460.

\section{Références}

BENNETT CLARK S., 1979. Mucosal coenzyme A dependent cholesterol after intestinal perfusion of lipids in rats. J. biol. Chem., 254, 1534-1536.

BOUCROT Ph., 1979. Prépondérance des phosphatidylcholines par rapport aux lysophosphatidylcholines dans les micelles de l'intestin chez le rat. J. Physiol., 75, 911-918.

FOLCH J., LEES M., SLOANE-STANLEY G. H., 1957. A simple method for the isolation and purification of total lipids from animal tissues. J. biol. Chem., 226, 497-509.

DESCARGUES G., 1975. Etude des triglycérides de l'huile d'olive. Th. Doct.-Ing., Dijon, France.

NALBONE G., LAIRON D., LAFONT H., DOMINGO N., HAUTON J. C., 1974. Behaviour of biliary phospholipids in intestinal lumen during fat digestion in rat. Lipids, 9, 765-770.

OLSSON O., BOUCROT Ph., 1974. Résistance des phospholipides endogènes intraluminaires à I'hydrolyse pancréatique chez le rat intact. Arch. inf. Physiol. Biochim., 82, 679-699.

RAGHAVAN S. S., GANGULY J., 1967. Studies on the intestinal absorption of triglycerides and fatty acids in rats. Ind. J. Biochem., 4, 68-74.

REISSER D., BOUCROT Ph., 1978. In vitro and in vivo effects of exogenous lipids on the enzymatic hydrolysis of rat bile phospholipids. Lipids, 13, 796-800.

ULRICH C., BOUCROT Ph., CLÉMENT J., 1974. Site of biliary phospholipid absorption in the small intestine of the rat. Biochimie, 56, 429-434. 